\title{
Aggregates of Diketopyrrolopyrrole Dimers in Solution
}

\author{
Robert Wawrzinek $^{\mathrm{a}, \mathrm{b}, *}$ Xiuwen Zhou, $^{\mathrm{a}, \mathrm{b}}$ Mujeeb Ullah, ${ }^{\mathrm{a}, \mathrm{b}}$ Ebinazar B. Namdas $^{\mathrm{a}, \mathrm{b}, *}$ and Shih- \\ Chun Lo ${ }^{\mathrm{a}, \mathrm{c}, *}$ \\ ${ }^{a}$ Centre for Organic Photonics \& Electronics, The University of Queensland, \\ ${ }^{b}$ School of Mathematics and Physics, ${ }^{c}$ School of Chemistry and Molecular Biosciences, \\ Brisbane, Queensland 4072, Australia.
}

\section{Abstract}

Dimers based on diketopyrrolopyrrole (DPP) chromophores have gained tremendous interest as an excellent material building block for organic solar cells and photodiodes. However, a counterintuitively blue shift in their solution absorption spectra of DPP chromophores occurs with an increasing number of thiophene bridging units. We allocate this as chromophore aggregates in solution, which might hinder adequate mixing in the blends, leading to poor film forming quality and reduced charge generation in solution processed devices. Hence, identification of such aggregates is crucial in order to find measures for device optimisation. In this study, we present synthesis and characterisation of three pyridyl end-capped DPP dimers of different conjugation length using thiophene linkers and compare their parent monomer to evidence the behaviours of aggregates in solution. We employ conventional and temperature dependent UV-Vis spectroscopy, fluorescence and excitation-emission spectroscopy as well as TD-DFT calculations to show that such DPP dimers predominantly form aggregates in solution even at low concentrations. By disentangling the spectroscopic behaviour of both aggregated and non-aggregated species, we refute literature's explanation that the apparent blue shift in absorption arises from a reduced conjugation length due to more molecular flexibility. Instead, absorption and emission signals of non-aggregated dimers are mostly masked by their aggregated species. This work provides a tool set using common laboratory spectroscopic equipment to identify and characterise solution aggregates-information particularly important towards optimisation of organic electronics processed from solution. 
Keywords: aggregation, organic electronics, diketopyrrolopyrroles, fluorescence, spectroscopy 


\section{Introduction}

Organic semiconductors have shown the great potential for low-cost, light-weight and largearea organic electronics. ${ }^{[1]}$ In fabricating the active organic semiconductor layer in organic electronics, both thermal deposition and solution process have been generally employed, where the latter offers excellent prospects for more economic, lower temperature and faster manufacturing. In solution processed organic opto-electronics such as organic lightemitting diodes (OLEDs), organic solar cells (OSCs) and organic photodiodes (OPDs), the active layer typically consists of a blend of appropriate guest/host or donor/acceptor molecules, co-dissolved in an organic solvent and subsequently deposited on a substrate using such as spin-coating to form a guest-host (in OLEDs) or a bulk heterojunction (in OSCs and OPDs) layer. For more details of organic semiconductors and their applications, the reader can refer to recent review articles. ${ }^{[2 a-g]}$

One well-known class of active organic semiconducting molecules is diketopyrrolopyrroles (DPPs), which were originally used as dyes in paints and inks. Their outstanding performance as an electron donor in OSCs or OPDs has made them as one of the most commonly studied molecular scaffolds. ${ }^{[3-5]}$ Not only $\pi$-conjugated polymers but also small molecules based on DPPs have been synthesised and shown to exhibit excellent device performance over last decades. ${ }^{[6]}$ Unlike polymers that are prone to have batch-to-batch variation in molecular weight and polydispersity (and hence properties), small molecules can be synthesised in high purity via reproducible synthetic routes. However, device performance of many small molecules still has not matched with those of their polymeric counterparts. As a compromise, $\pi$-conjugated dimers of such as DPP chromophores have recently gained significant interest due to their aforementioned high reproducibility in material synthesis and good device performance, approaching to those of polymeric chromophores due to their extended $\pi$-conjugation. ${ }^{[7-15]}$

Interestingly, thiophene bridged DPP dimers show an unusual spectroscopic behaviour: an extension of the thiophene bridge results in an unusual hypsochromical shift in the solution absorption spectrum. This intuitively phenomenon has often been ascribed to an enhanced freedom of rotation with the increased number of thiophene units, leading to an 
interruption of effective conjugation length. ${ }^{[12,14,16]}$ However, this explanation contradicts the general understanding for the effect of $\pi$-conjugation length on spectroscopic behaviours. Moreover, a recent study by Fritzner et al. on the influence of thiophenes between 1-(1,1-dicyanomethylene)-cyclohex-2-ene (DCC) units has clearly showed that the expected bathrochromic shift in absorption is actually observed for their materials with longer thiophene bridges. ${ }^{[17]}$ We think that the unusual absorption behaviour of DPP dimers, in fact, arises from the formation of strong aggregates in solution. ${ }^{[8]}$

Although strong intermolecular interaction within an active bulk hetero-junction (BHJ) layer is desirable, formation of solution aggregates prior to the deposition often hinders adequate intermixing and results in poor film forming quality. Moreover, these aggregates can form clusters larger than the typical diffusion length of excitons in organic photovoltaics $(\approx 5-10$ $\mathrm{nm})$, drastically reducing charge dissociation efficiencies in the devices. ${ }^{[18]}$ This is due to a lower probability of excitons reaching the donor-acceptor interface within the $\mathrm{BHJ}$, hence reducing charge separation. In order to optimise device performance, identification of solution aggregates is of significance. This will lead to new required strategies (such as heating, additives, sonication etc.) to break them up in a solution phase prior to solution process (e.g., using spin-coatings).

Herein, we report the synthesis of a monomer and three dimers based on pyridyl endcapped bisthiophenyl DPP chromophore, bridged with different number of thiophene units (i.e., DPP 1 and 2-4TPDPP, respectively, as shown in Figure 1) and studies of their unique solution aggregate behaviour. By using conventional and temperature dependent UV-Vis, steady-state and time resolved photoluminescence spectroscopies as well as theoretical calculations, we disentangle their aggregated and non-aggregated photophysics in solution. In order to further elucidate the nature of the aggregates in question, we compare the dimers to their monomeric counterpart 1 (Figure 1).

This work also introduces excitation-emission spectroscopy as a valuable characterisation technique for organic semiconducting materials. Furthermore, it provides insight into a new strategy to identify solution aggregate phenomena as well as how to evaluate the 
spectroscopic behaviour of both aggregated and non-aggregated species in order to take measures towards future device optimisation.

\section{Results and discussion}

\section{i) Synthesis of the DPP monomer and dimers}

The dimeric molecules we chose for this study comprise of DPP moieties, linked by thiophene units and end-capped with electron-deficient pyridines to create a more pronounced donor-acceptor alternation (A-D-A-D-A-D-A) within the chromophore. The synthetic route to the monomer 1, and dimers 2TPDPP, 3TPDPP and 4TPDPP is outlined in Scheme 1. First, monomer 1 was prepared in a good yield of $85 \%$ via a palladium $\left[\mathrm{Pd}\left(\mathrm{PPh}_{3}\right)_{4}\right]$ catalysed Stille cross-coupling reaction of a known bromo-DPP 2 with excess of 2-(tri- $n$ butylstannyl)pyridine 3 . The non-symmetric DPP precursor 4 was obtained in a moderate yield by a reaction of $\mathbf{2}$ and $\mathbf{3}$ in a 1:1 molar ratio under the same Stille cross-coupling conditions. Homo-coupling of 4 with bis(tri-n-butyltin) gave 2TPDPP in a 58\% yield. To add additional thiophene units between the DPP cores, Stille cross-coupling of 4 with 2,5-bis(trin-butylstannyl)thiophene $\mathbf{5}$ or 5,5'-bis(tri- $n$-butylstannyl)-2,2'-bithiophene 6 was performed to give 3TPDPP and 4TPDPP, respectively, in moderate to good yields (Scheme 1). Precursor $\mathbf{2}$ as well as the tin compounds $\mathbf{3}$ and $\mathbf{6}$ were obtained by following literature procedures, while $\mathbf{5}$ is commercially available. ${ }^{[19-22]}$

All synthesised materials exhibited good thermal stability with decomposition temperatures (5\% weight loss) above $350{ }^{\circ} \mathrm{C}$. More details of their thermal as well as electrochemical properties can be found in the Supplementary Information.

\section{ii) UV-Vis spectroscopy}

Figure 2 shows solution absorption spectra of the new DPPs in chloroform. The high energy absorption bands around $350 \mathrm{~nm}$ can be primarily attributed to the pyridyl moieties and absorption between $400-450 \mathrm{~nm}$ to the thiophene bridges. ${ }^{[23]}$ The two dominant absorption peaks at $565 \mathrm{~nm}$ and $602 \mathrm{~nm}$ of DPP 1 arise from intramolecular charge transfer (ICT) transitions (i.e., 0-1 and 0-0 transitions, respectively). ${ }^{[2]}$ Similarly, the broad absorption bands between $500-800 \mathrm{~nm}$ of the DPP dimers (2-4TPDPP) can also be mainly assigned to 
ICT transitions of the DPP chromophores even though only 2TPDPP seems to exhibit distinct transition features. Compared to their parent monomer 1, a significant red-shift in the absorption for all three dimers is observed. This is expected due to their extended $\pi$-conjugation. However, a closer look at the dimers' absorption spectra shows an unusual hypsochromic shift occurring with the increasing number of thiophene units in 3TPDPP and 4TPDPP. We believe this arises from aggregates of the DPP dimers, leading to the red-shifted absorption and masking the absorption bands of the non-aggregated molecules. Within these aggregates, the intermolecular interactions become weaker with increasing the numbers of the thiophene bridges, bringing about the observed blue-shift in the absorption spectra. This agrees with those of reported self-assembled nanoaggregates of donor-acceptor small molecules with planar conformations in solution. ${ }^{[25,26]}$

Concentration dependent UV-Vis and emission spectroscopies did not show any significant changes in the shape of the spectra (see Figures S3-S5 in the Supplementary Information). A series of temperature dependent UV-Vis absorption measurements, however, revealed distinct differences between the monomer and dimers (Figure 3). While monomer 1 only exhibits an intensity decrease of its lowest energy absorption bands at elevated temperatures, all dimers additionally show a significant blue shift of the latter, which is similar to those of DPP polymers. ${ }^{[27,28]}$ 2TPDPP shows its transition features much more pronounced in toluene than in chloroform. It is noted that the ratio between these transitions does not change in the case of DPP 1 whereas 2TPDPP's 0-0 transition decreases more than its 0-1 transition. Qian et al. ascribed this to different aggregate motifs, where a stronger repulsion between sulphurs in adjacent molecules forces the aggregate to a slipped (J-type) rather than face-to-face (H-type) packing (see Figure S6 in the Supplementary Information). ${ }^{[24,25]}$ With increasing temperatures, this causes a reduction of intermolecular charge transfer due to a dispersal of aggregates. Face-to-face packing (DPP 1), however, results in absorption predominantly by intramolecular charge transfer and therefore shows only little change in the absorption peaks. Dimers 3TPDPP and 4TPDPP do not show distinct transition features but exhibit, like 2TPDPP, a blue shift in their absorption peaks at higher temperatures. It is noteworthy that even at elevated temperatures $\left(80^{\circ} \mathrm{C}\right)$, these blue shifted peaks $(650-700 \mathrm{~nm})$ do not completely disappear, indicating very strong 
intermolecular interaction. This would in fact explain why concentration dependent UV-Vis experiments did not show any spectral changes.

Figure 3 also provides absorption peaks, resulted from subtracting the absorbance of lower from higher temperatures (dashed lines). These peaks represent the position (not any quantitative value!) of the aggregates' low energy absorption. The fact that the peak positions are even further red-shifted than those of measured at room temperature suggests that none of the DPPs exclusively exists in their aggregated form. Moreover, a significant blue-shift in the peak of aggregates takes place with enlargement of the thiophene bridge (see Figure $\mathbf{S 8}$ in the Supplementary Information). We assign this to a decrease in intermolecular overlap between donor and acceptor motifs within the J-type aggregates, hence, reduced intermolecular packing. This trend is also reflected in solid state absorption spectra recorded from neat films (see Figures S9 and S10 in the Supplementary Information).

\section{iii) Steady-state and time-resolved photoluminescence}

To characterise new chromophores, it is mandatory to determine their emission properties. This is often performed by simply exciting the lowest energy absorption maximum (derived from UV-Vis measurements) and detecting the resulted emission intensity over a spectral range at longer wavelengths. In the case of monomer 1 , this excitation takes place at 600 $\mathrm{nm}$ and results in two main emission peaks at $633 \mathrm{~nm}$ and $683 \mathrm{~nm}$, respectively (Figure 4a). The solution photoluminescence quantum yield (PLQY) was determined to be $41 \%$. In contrast, exciting the dimers 2-4TPDPP at their longest absorption maxima not only gave very weak emission (with PLQYs below 1\%) but the emission maxima at lower energy. This suggests that the observed emission is not related to the chosen excitation wavelength (i.e., at their longest absorption maxima). To illustrate this, Figure 4 shows solution emission spectra of monomer 1 and dimers 2-4TPDPP at different excitation wavelength. As one can see, excitation of 1 at its absorption peak (600 nm) indeed gives the highest emission intensity whereas excitation at higher or lower excitation wavelengths results in a decrease of emission intensity. All three dimers, on the other hand, show their strongest emission intensities at excitation wavelengths lower than their dominant absorption maxima. 
It seems that the absorption maxima resulted in the dimers' emission must be hidden underneath different dominant, red-shifted absorption bands, which mostly result in nonradiative decay. We, therefore, assume that all detected emission for the DPP dimers arises from their non-aggregated species and is predominantly re-absorbed by the red-shifted absorption of aggregates. This is also reflected to the poor PLQYs of the dimers. Moreover, using time correlated single photon counting (TCSPC) measurements gave bi-exponential radiative decays for all compounds, which is expected when aggregation takes place (Figure S21 in the Supplementary Information). The averaged luminescence lifetimes show a decrease with the extension of the conjugation length and are given in Table 1. It needs to be stressed that PLQY measurements of isolated, non-aggregated DPP dimers are not accessible without exciting the omnipresent non-radiative aggregates. Hence, calculations of radiative lifetimes using the Strickler-Berg relation would not be valid. ${ }^{[29]}$

\section{iv) Steady-state excitation-emission spectroscopy}

To investigate what the actual absorption of non-aggregated molecules would look like, we performed (3D-) excitation-emission spectroscopy, a powerful technique usually used in petroleum and water analytics, research on museum artefacts and other areas where identification of different emissive species in complex mixtures is of interest. ${ }^{[30-35]}$ Herein single emission spectra for different excitation wavelength are recorded in a range of 300$700 \mathrm{~nm}$ and the results are $\mathrm{x}-\mathrm{y}$ contour plotted with emission intensity in z-direction. Figure 5 shows the results exemplarily for monomer 1 and dimer 2TPDPP (see Figures S15 and S16 for dimers 3TPDPP and 4TPDPP, respectively, in the Supplementary Information). To illustrate our interpretation of the results, we added conventional absorption and emission spectra (solid lines) as well as the intensity profiles (dashed lines) next to the $y$ - and $x$-axis, respectively (Figure 5 and Figures S17 \& S18 in the Supplementary Information).

In case of monomer $\mathbf{1}$, emission peaks only occur at excitation wavelengths matching with its corresponding conventional UV-Vis spectrum. In contrast, 2TPDPP shows a very different behaviour, where emission arises from excitation approximately between $500 \mathrm{~nm}$ and 650 $\mathrm{nm}$ (dashed line on $\mathrm{y}$-axis) rather than between $\approx 650-800 \mathrm{~nm}$, as in its conventional UV-Vis spectrum (solid line on y-axis). This supports our hypothesis that dimers 2-4TPDPP exist in 
solution predominantly as one or more aggregate species, which do not show and/or even heavily quench any emission. Furthermore, it is noteworthy that excitation-emission spectra of monomer 1 and dimers 2-4TPDPP generally resemble each other in shape but show differences in position of the peaks (Figure S19). It is evident that excitation leading to emission shifts towards longer wavelengths with increasing number of thiophene units (Figure S19, Supplementary Information).

This trend becomes more obvious in the classic 2D excitation spectra as shown in Figure 6a. The emission spectra of monomer and dimers in Figure $6 \mathrm{~b}$ were corrected by taking the inner filtering effect of the measured solution into account. ${ }^{[36 a]}$ Assuming that all detected dimer emission arises from non-aggregated molecules within the solution, these experiments clearly support the originally expected trend that extension of the $\pi$ conjugation indeed result in bathochromically shifted absorption.

\section{v) Solution aggregation and molar extinction coefficient}

At this point, it needs to stress that calculation of molar extinction coefficients for the nonaggregated dimer is difficult as the relevant absorption maxima are masked by their aggregated species and therefore not accessible for quantification. Ignoring this fact (and assuming identical absorption efficacy) would give $\varepsilon$ values of $33.9 \times 10^{3} \mathrm{~L} \mathrm{~mol}^{-1} \mathrm{~cm}^{-1}$ for monomer 1 and 82.6-85.7 $\times 10^{3} \mathrm{~L} \mathrm{~mol}^{-1} \mathrm{~cm}^{-1}$ for the dimers at their longest wavelength absorption peaks in dichloromethane (see Figure S2 in the Supplementary Information). These values are, in fact, higher than the expected doubling from having two DPP units in the material when comparing the dimers to monomer 1 . This again indicates the presence of different species in the solution (the difference in thiophene units is expected to have a negligible effect on the $\varepsilon$ ). Interestingly, such similarity between $\varepsilon$ values throughout the dimer series also indicates that the blue shift of the dimer aggregates' absorption is not caused by the amount of aggregates but predominantly by the intensity of intermolecular interaction within them. The latter obviously decreases when going from 2TPDPP to 4TPDPP, as reflected in smaller differences between absorption spectra of different aggregation states (solution non-aggregated, solution aggregated, solid-state. See Figure S10, 
Supplementary Information). The spectroscopic properties of monomer $\mathbf{1}$ and dimers 2-4TPDPP are summarised in Tab. 2.

\section{vi) Theoretical calculations}

To better understand the nature of the observed absorption bands, we performed TD-DFT calculations with Gaussian 09 code at B3LYP/6-31G** level for optimised ground-state structures of monomer 1 and dimers 2-4TPDPP (Figure S22 in the Supplementary Information). ${ }^{[37-40]}$ The calculation showed a red shift for the dimers in absorption by approximately $0.4 \mathrm{eV}$ (from $2.09 \mathrm{eV}$ to $\approx 1.67 \mathrm{eV}$ ) with respect to the monomer 1 . Increasing the number of thiophene bridging units causes only subtle differences in absorption wavelength (or $\approx 0.01 \mathrm{eV}$ ) but a fair increase in absorption peak intensity (Figure S23 in Supplementary Information). Our theoretical results are in agreement with the trend of the change in absorption induced by dimers from the work by Stas et al., where similar monomer and three DPP dimers without pyridyl end-caps were studied. ${ }^{[12]}$ Their calculated absorption energies of $2.15 \mathrm{eV}, 1.77 \mathrm{eV}, 1.74 \mathrm{eV}, 1.73 \mathrm{eV}$ for monomer and dimers with 2-4 thiophenes within the dimer bridge, respectively, also show a subtle red shift of $\approx 0.4 \mathrm{eV}$ with the increase of thiophene units but as one can see these energy values already converge. The pyridyl moieties in our DPPs are expected to stablise the energy levels of the conjugated systems even further and can explain why the obtained absorption energies for all three dimers are within the calculation error.

\section{Conclusion}

We have synthesised and characterised a series of pyridine end-capped bisthiophenyl DPPs monomer and dimers with two, three and four thiophene bridging units. Using a combination of spectroscopic techniques as well as theoretical calculations, we were able to elucidate why conventional UV-Vis spectroscopy of such dimers show a hypsochromic shift in solution absorption spectra with increasing number of thiophene units. Supposedly, DPP dimers form non-emissive (J-type-) aggregates in solution, masking their absorption maxima of their emissive non-aggregated species. This results in drastic fluorescence quenching of the latter. Hence, much lower photoluminescence quantum yields were observed when compared to their $\mathrm{H}$-aggregates. Furthermore, we disentangled the spectroscopic 
properties of aggregated and non-aggregated DPPs in solution and assigned the initially blue shift observed in solution absorption to the weakened intermolecular interaction between aggregates with increased number of bridging thiophene units. Measurements on the nonaggregated DPP dimers indeed show the expected red-shifts in solution absorption and emission. This debunks an idea of hypsochromic shifts in absorption due to a disrupted $\pi$ conjugation caused by increased molecular flexibility.

This study also introduces excitation-emission spectroscopy as a valuable tool for organic electronics to characterise aggregation behaviour of photoactive materials in solution. Although strong intermolecular interactions are generally desirable in solid state, solution aggregation hinders adequate mixing in the blends, which may well cause poor film forming quality. Hence, detailed information on aggregation behaviour (especially for fabrication of solution processed devices) is crucial and can be used to find methods to manipulate the formation of such aggregates in order to optimise performance of organic electronic devices.

\section{Acknowledgements}

We thank the Australian Research Council (ARC) for financial support (ARC DP140101088). We also thank Dr. Dani M. Stoltzfus for performing the PESA measurements, Dr. Paul E. Shaw for helpful discussion, and Graham MacFarlane and Peter Josh for mass spectrometry measurements. E.B.N. is a recipient of a UQ Fellowship. X.Z. is a recipient of an Early Postdoc Mobility Fellowship from Swiss National Science Foundation (P2GEP2_155709). This research was undertaken with the assistance of resources provided at the $\mathrm{NCl}$ National Facility systems at the Australian National University through the National Computational Merit Allocation Scheme supported by the Australian Government, including grant LE120100181.

\section{Supplementary Information}

General methods, synthetic procedures, and various experimental and photophysical details.

\section{References}


[1]

M. V. Jacob, Electronics, 2014, 3, 594.

[2]

a) M. Gsänger, D. Bialas, L. Huang , M. Stolte and F. Würthner, Adv. Mater., 2016, 28, 3615.

b) C. B. Nielsen, S. Holliday, H.-Y. Chen, S. J. Cryer and I. McCulloch, Acc. Chem. Res., 2015, 48, 2803.

c) W. Ni, X. Wan, M. Li, Y. Wang and Y. Chen, Chem. Commun., 2015, 51, 4936.

d) L. Dou, Yo. Liu, Z. Hong, G. Li and Y. Yang, Chem. Rev., 2015, 115, 12633.

e) A. Broggi, I. Tomasi, L. Bianchi, A. Marrocchi and L. Vaccaro, ChemPlusChem, 2014, 79, 486.

f) Y. Li, P. Sonar, L. Murphy and W. Hong, Energy Env. Sci., 2013, 6, 1684.

g) R. D. Jansen-van Vuuren, A. Armin, A. K, Pandey, P. L. Burn and P. Meredith, Adv. Mater., 2016, 28, 4766.

[3]

Z. Hao and I. Abul, Chem. Soc. Rev., 1997, 26, 203.

[4]

S. Qu and H. Tian, Chem. Commun., 2012, 48, 3039.

[5]

Y. Li, P. Sonar, L. Murphy and W. Hong, Energy Environ. Sci., 2013, 6, 1684.

[6]

B. Walker, A. B. Tamayo, X.-D. Dang, P. Zalar, J. H. Seo, A. Garcia, M. Tantiwiwat and T.-Q. Nguyen, Adv. Funct. Mater., 2009, 19, 3063.

[7]

S. Loser, C. J. Bruns, H. Miyauchi, R. P. Ortiz, A. Facchetti, S. I. Stupp and T. J. Marks, J. Am. Chem. Soc., 2011, 133, 8142.

[8]

J. W. Lee, Y. S. Choi and W. H. Jo, Org. Electron., 2012, 13, 3060.

[9]

D. Credgington, F. C. Jamieson, B. Walker, T.-Q. Nguyen and J. R. Durrant, Adv. Mater., 2012, 24, 2135. 
J. Huang, C. Zhan, X. Zhang, Y. Zhao, Z. Lu, H. Jia, B. Jiang, J. Ye, S. Zhang, A. Tang, Y. Liu, Q. Pei and J. Yao, ACS Appl. Mater. Interfaces, 2013, 5, 2033.

Y. S. Choi and W. H. Jo, Org. Electron., 2013, 14, 1621.

S. Stas, J.-Y. Balandier, V. Lemaur, O. Fenwick, G. Trenago, F. Quist, F. Cacialli, J. Cornil and Y. H. Geerts, Dyes Pigm., 2013, 97, 198.

C. Lu and W.-C. Chen, Chem. Asian J., 2013, 8, 2813.

T. R. Hong, J. Shin, H. A. Um, T. W. Lee, M. J. Cho, G. W. Kim, J. H. Kwon and D. H. Choi, Dyes Pigm., 2014, 108, 7.

\section{[15]}

D. Yu, Y. Liu. M. Xiao, Q. Fan, W. Su, X. Li, H. Tan, Y. Wang, R. Yang, and W. Zhu, Dyes Pigm., 2016, 125, 151.

M. S. Chen, J. R. Niskala, D. A. Unruh, C. K. Chu, O. P. Lee and M. J. Fréchet, Chem. Mater., 2013, 25, 4088.

[17]

R. Fitzner, E. Mena-Osteritz, K. Walzer, M. Pfeiffer and P. Bäuerle, Adv. Funct. Mater., 2015, 25, 1845.

O. V. Mikhnenko, M. Kuik, J. Lin, N. van der Kaap, T.-Q. Nguyen and P. W. M. Blom, Adv. Mater., 2014, 26, 1912.

\section{[19]}

Y. Li, S. Singh and P. Sonar, Adv. Mater., 2010, 22, 4862.

[20]

T. N. Y. Hoang, M. Humbert-Droz, T. Dutronc, L. Guénée, C. Besnard and C. Piguet, Inorg. Chem., 2013, 52, 5570. 
J. Huang, J. Miragliotta, A. Becknell and H. E. Katz, J. Am. Chem. Soc., 2007, 129, 9366.

[22]

E. V. Lukovskaya, A. A. Kosmacheva, O. A. Fedorova, A. A. Bobyleva, A. V. Dolganov, N. E. Shepel, Y. V. Fedorov, V. V. Novikov and A. V. Anisimov, Russ. J. Org. Chem., 2014, 50, 552.

A. Riano, P. M. Burrezo, M. J. Mancheno, A. Timalsina, J. Smith, A. Facchetti, T. J. Marks, J. T.

L. Navarrete, J. L. Segura, J. Casado and R. P. Ortiz, J. Mater. Chem. C, 2014, 2, 6376.

D. Qian, B. Liu, S. Wang, S. Himmelberger, M. Linares, M. Vagin, C. Müller, Z. Ma, S. Fabiano, M. Berggren, A. Salleo, O. Inganas, Y. Zou and F. Zhang, J. Mater. Chem. A, 2015, 3, 24349.

\section{[25]}

E. H. A. Beckers, S. C. J. Meskers, A. P. H. J. Schenning, Z. Chen, F. Würthner, P. Marsal, D. Bejonne, J. Cornil and R. A. J. Janssen, J. Am. Chem. Soc., 2006 128, 649.

[26]

Y. Zhou, C. X. Guzman, L. C. Heleguero-Kelley, C. Liu, S. R. Perifoy, B. Captain and A. B. Braunschweig, J. Phys. Org. Chem., 2016, 10.1002/poc.3548.

R. S. Ashraf, I. Meager, M. Nikolka, M. Kirkus, M. Planells, B. C. Schroeder, S. Holliday, M. Hurhangee, C. B. Nielsen, H. Sirringhaus and I. McCulloch, J. Am. Chem. Soc., 2015, 137, 1314.

[28]

B. C. Schroeder, S. Rossbauer, R. J. Kline, L. Biniek, S. E. Watkins, T. D. Anthopoulos, I. McCulloch and C. B. Nielsen, Macromolecules, 2014, 47, 2833.

S. J. Strickler and R. A. Berg, J. Chem. Phys., 1962, 37, 814.

[30]

D. Patra and A. K. Mishra, Anal. Bioanal. Chem, 2002, 373, 304.

J. B. C. Bugden, C. W. Yeung, P. E. Kepkay and K. Lee, Marine Poll. Bull., 2008, 56, 677. 
F. Herzi, N. Jean, A. S. Hlaili and S. J. Mounier, J. Phycol., 2014, 50, 665.

[33]

L. J. Soltzberg, S. Lor, N. Okey-Igwe and R. Newman, Am. J. Anal. Chem., 2012, 3, 622.

[34]

M. R. Van Bommel, I. V. Berghe, A. M. Wallert, R. Boitelle and J. Wouters, J. Chromatogr. A, 2007, 1157, 260.

[35]

R. Wawrzinek and P. Wessig, Dyes Pigm., 2015, 123, 39.

[36]

a) J. R. Lakowicz, Principles of fluorescence spectroscopy. Springer, New York, $3^{\text {rd }}$ Ed., 2006, 2, p56.

b) J. R. Lakowicz, Principles of fluorescence spectroscopy. Springer, New York, $3^{\text {rd }}$ Ed., 2006, 1, p7.

M. E. Casida, Time-dependent density functional theory for molecules, Springer, 1995, 1, p155.

[38]

M. J. Frisch et al., Gaussian 09, Revision E.01, Gaussian Inc., Wallingford, CT, 2009.

a) P. J. Stephens, F. J. Devlin, C. F. Chabalowski and M. J. Frisch, J. Phys. Chem., 1994, 98, 11623.

b) A. D. Becke, J. Chem. Phys., 1993, 98, 5648.

c) C. Lee, W. Yang and R. G. Parr, Phys. Rev. B: Condens. Matter Mater. Phys., 1988, 37, 785.

[40]

M. M. Francl, W. J. Pietro, W. J. Hehre, J. S. Binkley, D. J. DeFrees, J. A. Pople and M. S.

Gordon, J. Chem. Phys., 1982, 77, 3654. 

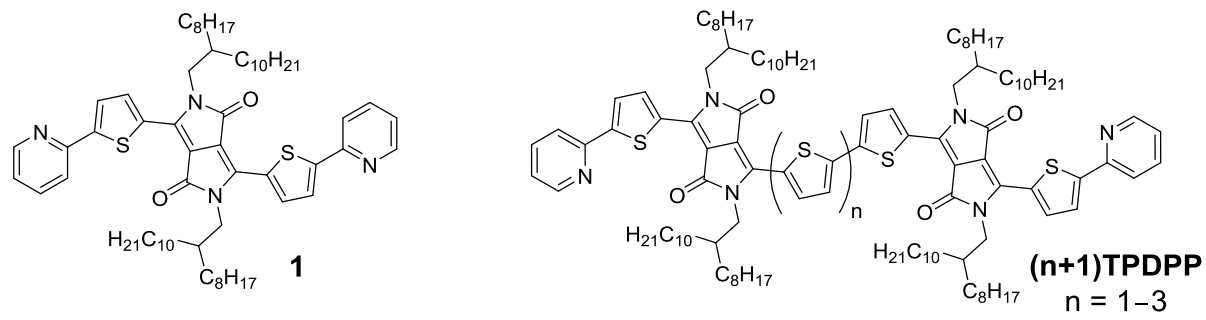

Figure 1. Chemical structures of DPP monomer 1, and dimers 2TPDPP, 3TPDPP \& 4TPDPP.

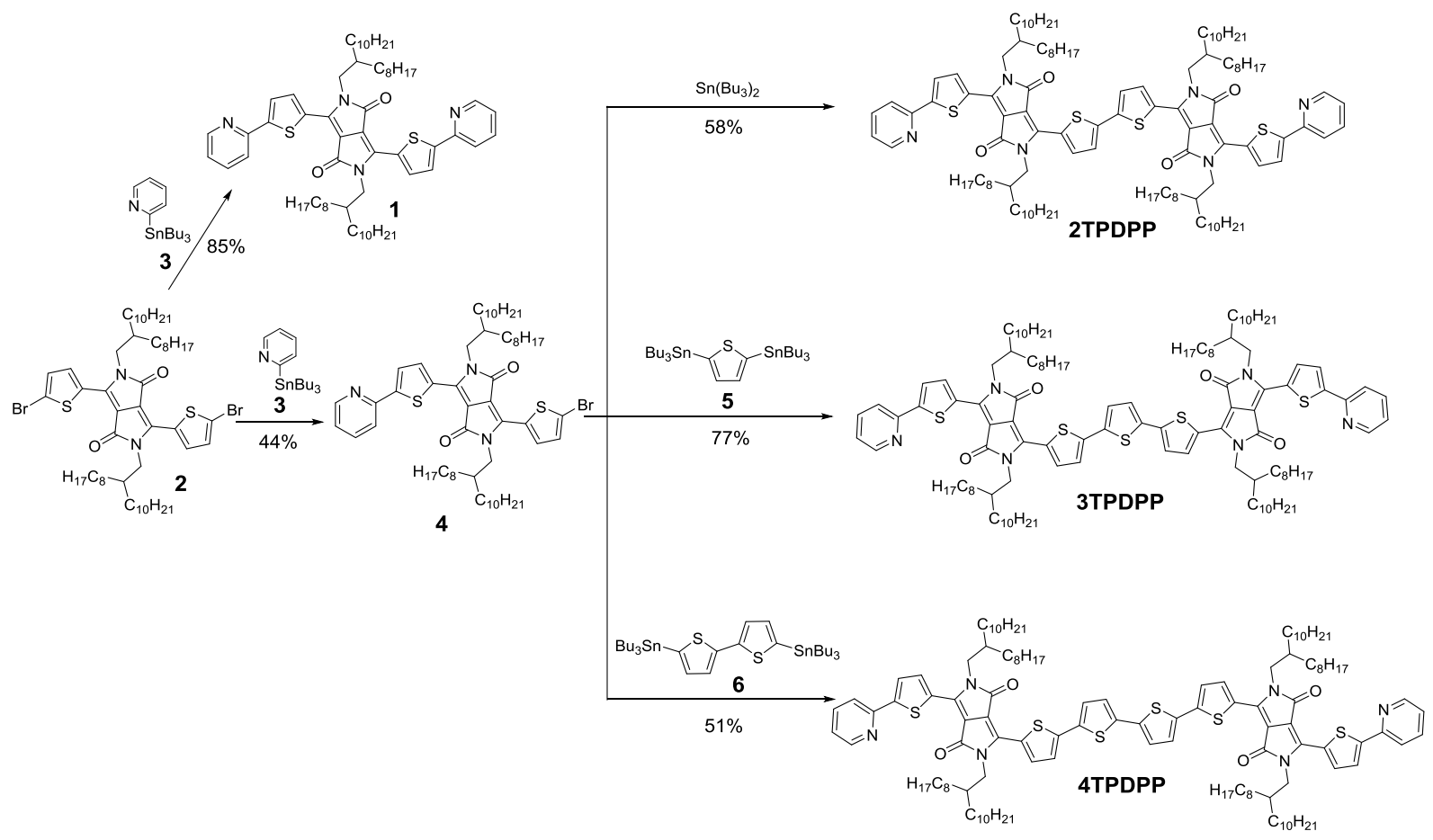

Scheme 1. Synthetic route to monomer 1 and dimers 2TPDPP, 3TPDPP \& 4TPDPP. 


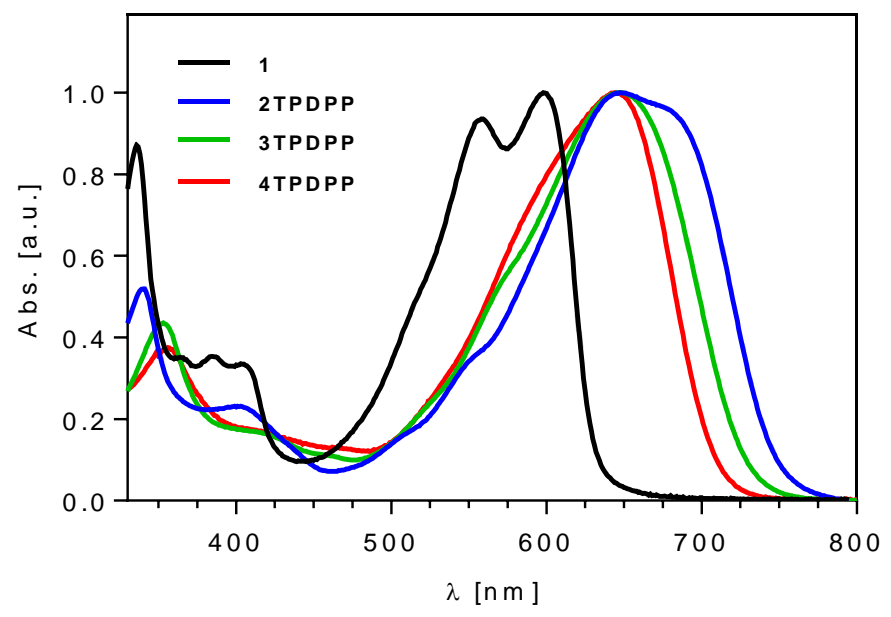

Figure 2. Normalised solution UV-Vis spectra of monomer 1 and dimers 2TPDPP, 3TPDPP, \& 4TPDPP (in chloroform).
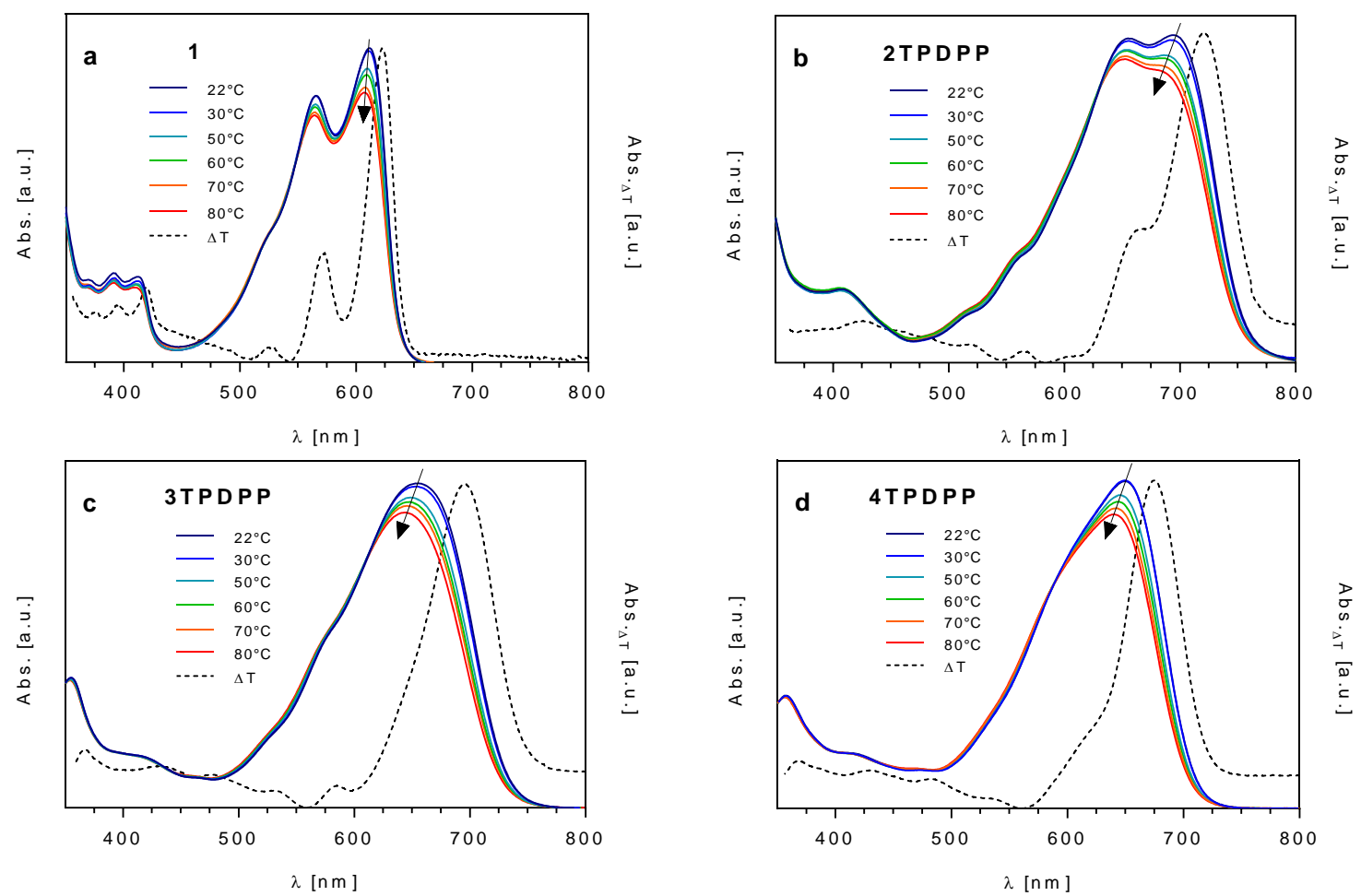

Figure 3. Temperature dependent UV-Vis absorption spectra of monomer $\mathbf{1}$ (a) and dimers 2TPDPP (b), 3TPDPP (c) \& 4TPDPP (d) in toluene $\left(22-80^{\circ} \mathrm{C}\right.$; for experimental details see Supplementary Information). The dashed lines represent the normalised difference in the absorption between higher and lower temperatures. 

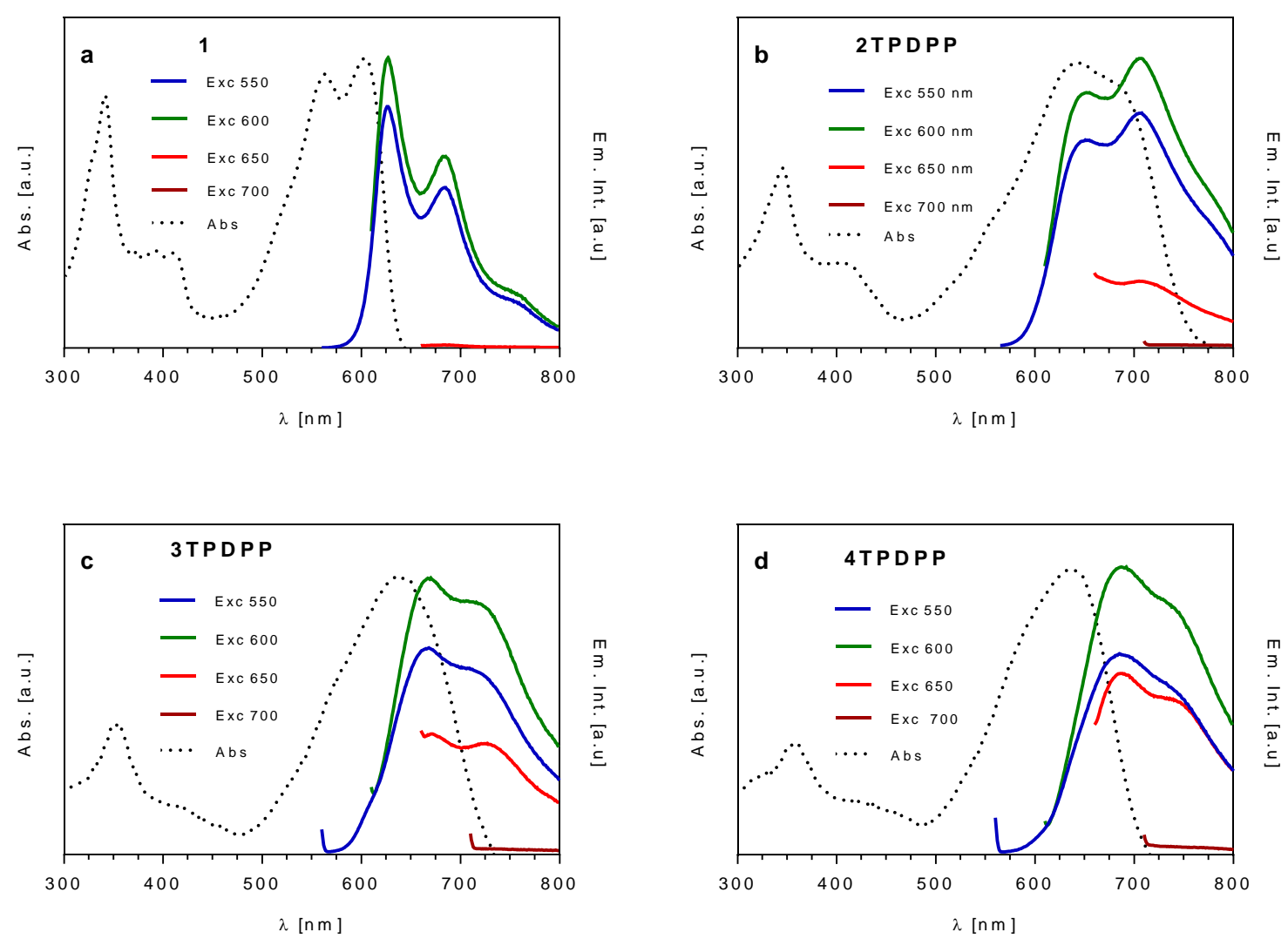

Figure 4. Uncorrected emission spectra of monomer 1 (a) and dimers 2TPDPP (b), 3TPDPP (c) \& 4TPDPP (d) in chloroform at different excitation wavelengths. The dashed lines represent their normalised absorption spectra in chloroform at room temperature. 


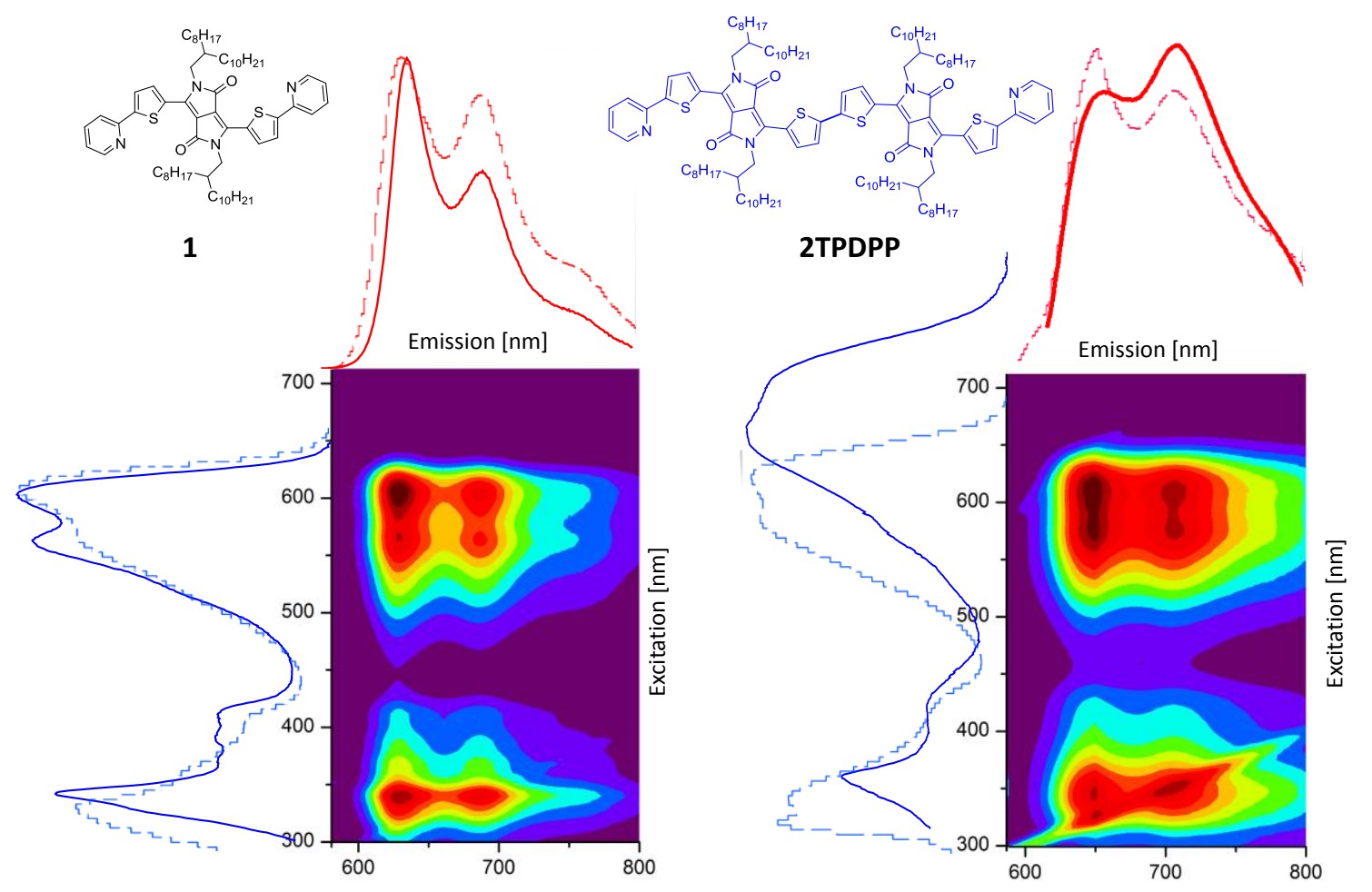

Figure 5. Excitation-emission spectra of monomer 1 and dimer 2TPDPP (in chloroform). Dashed lines are spectra taken from the excitation-emission profiles of Figure S17 in the Supplementary Information, and solid lines are from UV-Vis and steady-state fluorescence measurements. 

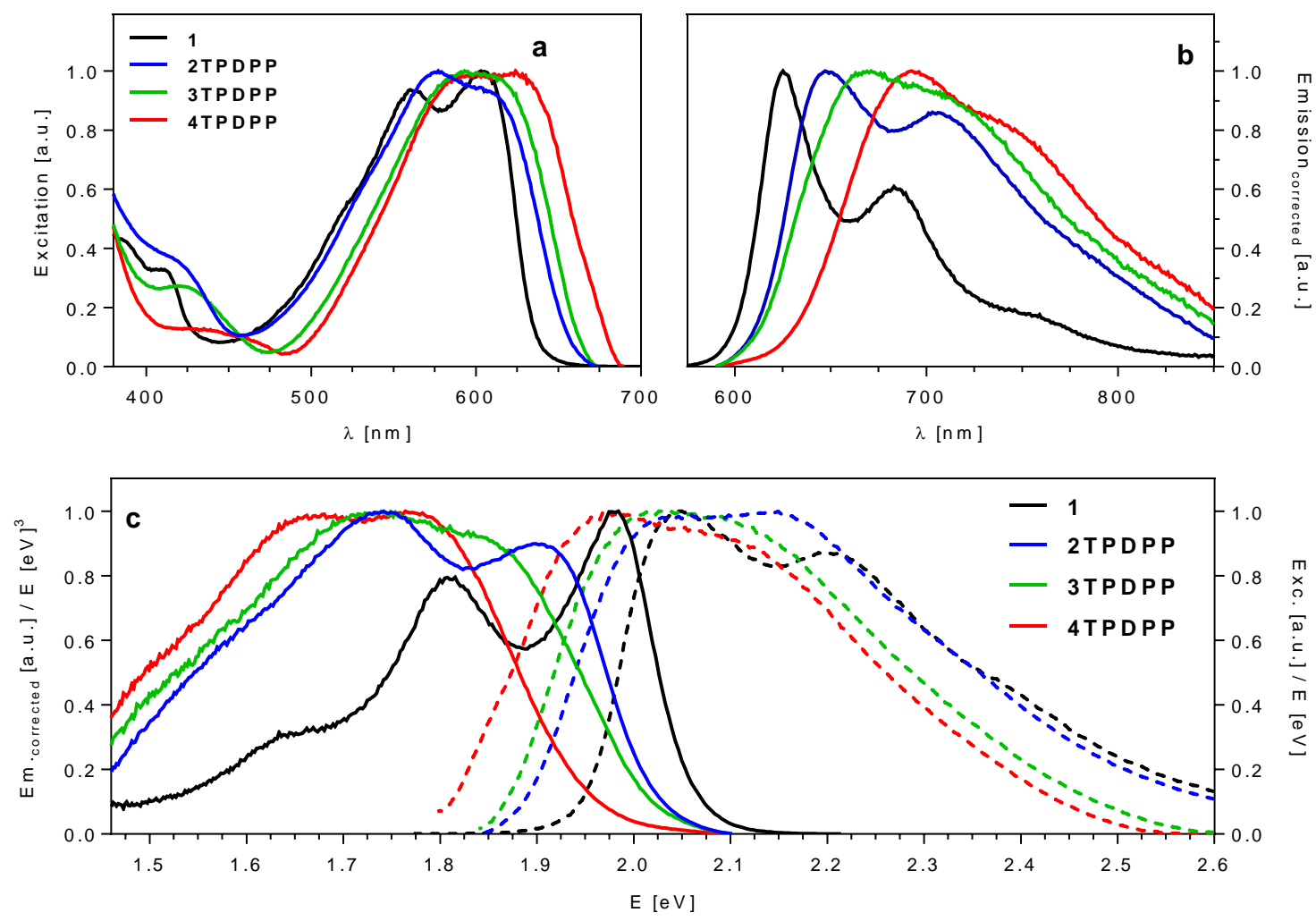

Figure 6. Excitation (a) and corrected emission spectra (b) of monomer 1 and dimers 2-4TPDPP in chloroform. The detection wavelengths in a) were $626 \mathrm{~nm}$ (for 1) and $650 \mathrm{~nm}$ (for 2-4TPDPP). c) Modified and normalised excitation and emission spectra plotted against $\mathrm{eV} .^{[36 \mathrm{~b}]}$

Table 1. Summary of optical properties of the DPPs.

\begin{tabular}{ccccccc}
\hline Compound & $\begin{array}{c}\lambda_{\text {abs }} \\
\text { theoretical } \\
{[\mathrm{eV}](\mathrm{nm})^{\mathrm{a}}}\end{array}$ & $\begin{array}{c}\lambda_{\text {abs }} \\
\text { aggregated } \\
{[\mathrm{nm}]^{\mathrm{b}, \mathrm{c}}}\end{array}$ & $\begin{array}{c}\lambda_{\text {abs }} \\
\text { non-aggreg. } \\
{[\mathrm{nm}]^{\mathrm{d}, \mathrm{e}}}\end{array}$ & $\begin{array}{c}\lambda_{\mathrm{em}} \\
\text { non-aggreg. } \\
{[\mathrm{nm}]^{\mathrm{d}}}\end{array}$ & $\begin{array}{c}\text { PLQY } \\
{[\%]^{\mathrm{d}}}\end{array}$ & $\begin{array}{c}\tau_{\text {avg }} \\
{[\mathrm{ns}]^{\mathrm{d}}}\end{array}$ \\
\hline 1 & $2.09(593)$ & 571,624 & 603 & 625,683 & 41 & 3.6 \\
2TPDPP & $1.67(742)$ & $664 \mathrm{sh}, 721$ & 608 & 648,706 & $<1$ & 2.1 \\
3TPDPP & $1.66(746)$ & 694 & 613 & $669,712 \mathrm{sh}$ & $<1$ & 1.5 \\
4TPDPP & $1.67(744)$ & 675 & 621 & $693,729 \mathrm{sh}$ & $<1$ & $<1$ \\
\hline
\end{tabular}

a TD-DFT [B3LYP/6-31G**]; ${ }^{b}$ in toluene; ${ }^{c}$ from temperature dependent difference in absorbance; ${ }^{d}$ in chloroform; ${ }^{e}$ estimated lowest energy peak or shoulder in excitation spectra. 


\title{
Aggregates of Diketopyrrolopyrrole Dimers in Solution
}

\author{
Robert Wawrzinek $^{\mathrm{a}, \mathrm{b}, *}$ Xiuwen Zhou, $^{\mathrm{a}, \mathrm{b}}$ Mujeeb Ullah, ${ }^{\mathrm{a}, \mathrm{b}}$ Ebinazar B. Namdas $^{\mathrm{a}, \mathrm{b}, *}$ and Shih- \\ Chun Lo ${ }^{\mathrm{a}, \mathrm{c}, *}$ \\ ${ }^{a}$ Centre for Organic Photonics \& Electronics, The University of Queensland, \\ ${ }^{b}$ School of Mathematics and Physics, ${ }^{c}$ School of Chemistry and Molecular Biosciences, \\ Brisbane, Queensland 4072, Australia.
}

Figure 1. Chemical structures of DPP monomer 1, and dimers 2TPDPP, 3TPDPP \& 4TPDPP.

Scheme 1. Synthetic route to monomer 1 and dimers 2TPDPP, 3TPDPP \& 4TPDPP.

Figure 2. Normalised solution UV-Vis spectra of monomer 1 and dimers 2TPDPP, 3TPDPP, \& 4TPDPP (in chloroform).

Figure 3. Temperature dependent UV-Vis absorption spectra of monomer $\mathbf{1}$ (a) and dimers 2TPDPP (b), 3TPDPP (c) \& 4TPDPP (d) in toluene $\left(22-80^{\circ} \mathrm{C}\right.$; for experimental details see Supplementary Information). The dashed lines represent the normalised difference in the absorption between higher and lower temperatures.

Figure 4. Uncorrected emission spectra of monomer 1 (a) and dimers 2TPDPP (b), 3TPDPP (c) \& 4TPDPP (d) in chloroform at different excitation wavelengths. The dashed lines represent their normalised absorption spectra in chloroform at room temperature.

Figure 5. Excitation-emission spectra of monomer 1 and dimer 2TPDPP (in chloroform). Dashed lines are spectra taken from the excitation-emission profiles of Figure S17 in the Supplementary Information, and solid lines are from UV-Vis and steady-state fluorescence measurements.

Figure 6. Excitation (a) and corrected emission spectra (b) of monomer 1 and dimers 2-4TPDPP in chloroform. The detection wavelengths in a) were $626 \mathrm{~nm}$ (for 1) and $650 \mathrm{~nm}$ (for 2-4TPDPP). c) Modified and normalised excitation and emission spectra plotted against $\mathrm{eV} \cdot{ }^{[36 \mathrm{~b}]}$ 


\section{Aggregates of Diketopyrrolopyrrole Dimers in Solution}

Robert Wawrzinek $^{\mathrm{a}, \mathrm{b}, *}$ Xiuwen Zhou, ${ }^{\mathrm{a}, \mathrm{b}}$ Mujeeb Ullah, ${ }^{\mathrm{a}, \mathrm{b}}$ Ebinazar B. Namdas $^{\mathrm{a}, \mathrm{b}, *}$ and ShihChun Lo ${ }^{a, c, *}$

\section{Graphical Abstract}

3D excitation-emission spectroscopy disentangles the spectroscopic properties of aggregates and non-aggregates of DPPs based dimers, Rertob Wawrzinek, Xiuwen Zhou, Mujeeb Ullah, Ebinazar B. Namdasa, and Shih-Chun Lo

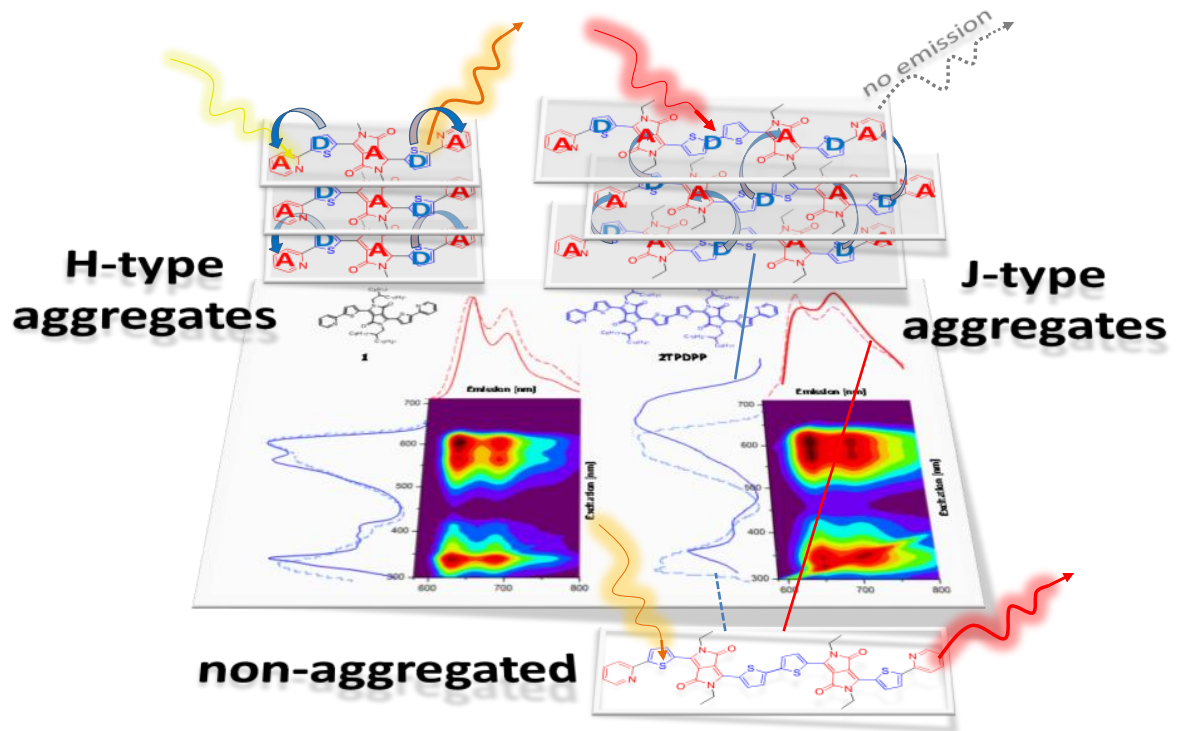

